\title{
Line profile variations in classical Cepheids
}

\section{Evidence for non-radial pulsations?^}

\author{
V. V. Kovtyukh ${ }^{1,2}$, S. M. Andrievsky ${ }^{1,2}$, R. E. Luck ${ }^{3}$, and N. I. Gorlova ${ }^{1,2,4}$ \\ 1 Department of Astronomy and Astronomical Observatory of Odessa State University, Shevchenko Park, 65014, \\ Odessa, Ukraine \\ 2 Isaac Newton Institute of Chile, Odessa Branch, Ukraine \\ 3 Department of Astronomy, Case Western Reserve University, 10900 Euclid Avenue, Cleveland, OH 44106-7215, USA \\ e-mail: luck@fafnir.astr.cwru.edu \\ ${ }^{4}$ Department of Astronomy, The University of Arizona, Tucson, AZ 85721, USA
}

Received 30 July 2002 / Accepted 14 January 2003

\begin{abstract}
We have investigated line profiles in a large sample of Cepheid spectra, and found four stars that show unusual (for Cepheids) line profile structure (bumps or/and asymmetries). The profiles can be phase dependent but the behavior persists over many cycles. The asymmetries are unlikely to be due to the spectroscopic binarity of these stars or the specific velocity field in their atmospheres caused by shock waves. As a preliminary hypothesis, we suggest that the observed features on the line profiles in the spectra of X Sgr, V1334 Cyg, EV Sct and BG Cru can be caused by the non-radial oscillations. It is possible that these non-radial oscillations are connected to resonances between the radial modes $(3.2,7.5$ or 10.0$)$.
\end{abstract}

Key words. stars: oscillations - stars: variables: Cepheids - stars: individual: X Sgr, V1334 Cyg, EV Sct, BG Cru

\section{Introduction}

The problem of line profile variation in classical Cepheids has been a subject of many studies, for example, Karp (1975a, 1975b), Butler et al. (1996). Generally speaking, the profile variations reflect the movements of the gas in radially pulsating atmosphere, and nowadays they are well understood in the framework of radiative hydrodynamics. The shifts of the line center mass and time-dependent profile asymmetry are characteristic features of all classical Cepheids. Along with this, some Cepheids were reported to demonstrate an unusual behaviour of their line profiles. Evidence of a strange doubling of a number of low-excitation lines in a classical Cepheid was firstly reported by $\mathrm{Kraft}$ (1956) for X Cyg $(P=16$ d 386). However, subsequent observations of this star by Butler (1993) and our data (20 spectra, unpublished) did not recover this effect. Kraft (1967), though, has shown that line doubling in X Cyg varies from cycle to cycle.

Later, Sasselov et al. (1989), and then Sasselov \& Lester (1990) investigated unusual structure in the line profiles of the Cepheid X Sgr. They noticed line doubling (splitting)

Send offprint requests to: V. V. Kovtyukh,

e-mail: val@deneb.odessa.ua

$\star$ Based on spectra collected at McDonald $2.1 \mathrm{~m}$ and Kitt Peak $4 \mathrm{~m}$ (USA), CTIO $4 \mathrm{~m}$ (Chile) and MSO $1.8 \mathrm{~m}$ (Australia). in X Sgr using infrared spectra. Figures from those papers clearly show the additional blue- or red-shifted absorptions in the line profiles. For instance, both blue and red absorptional components together with a central absorption are clearly seen at $\phi($ dyn $)=0.13$ (see Fig. 12 in Sasselov \& Lester 1990). The authors also pointed out the stability of this phenomenon during the 11 month period spanned by their observations. In 1990, Sasselov \& Lester made an attempt to interpret the phenomenon described by Sasselov et al. (1989) as a result of an entangled atmospheric velocity field, specifically by supposing the existence of pulsationally-driven shock-waves and radiative transfer along their path in the atmosphere. The main difficulty of such a rather loose interpretation is that while the additional absorption components at the line profiles are present over the whole period of pulsation, it is impossible for the shock waves to propagate continuously during the whole cycle.

In 1999, Kovtyukh \& Andrievsky detected anomalous line splitting (i.e. satellite absorption at the profile of each line) in the spectrum of another classical Cepheid, EV Sct, and interpreted it as a sign of spectroscopic binarity of this Cepheid. Kiss \& Vinkó (2000) discovered additional absorption components in the spectral lines of a classical Cepheid V1334 Cyg, and also attributed this phenomenon to a fourth component in the system of this Cepheid. However, such an interpretation of line splitting based on possible binarity suffers from some weak 


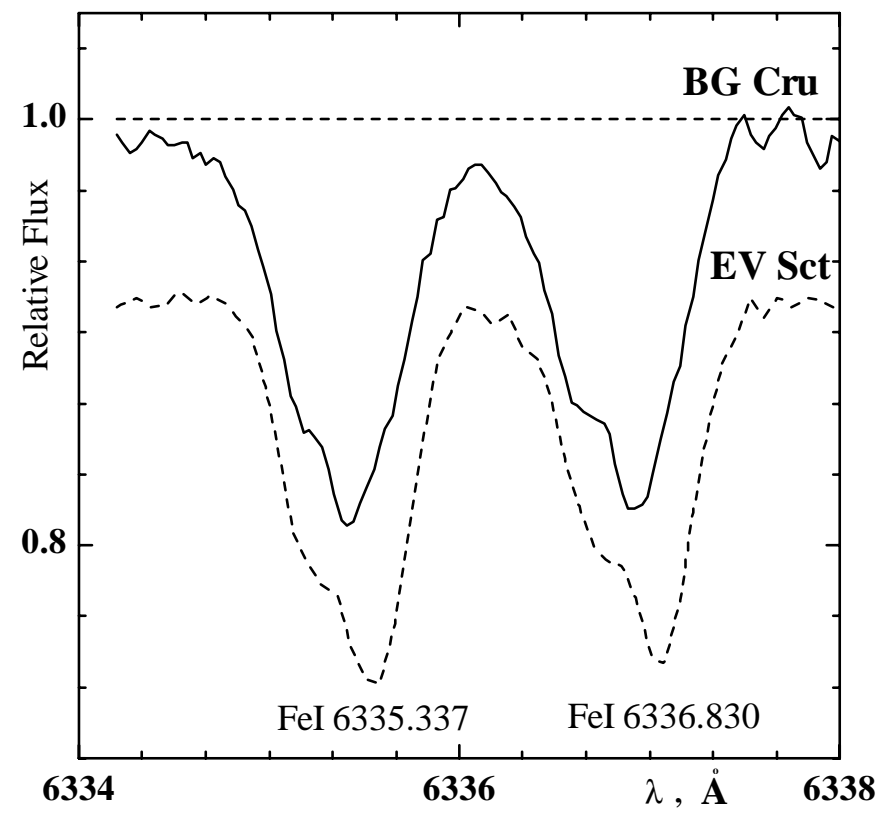

Fig. 1. Absorption bumps in the spectra of BG Cru $(\phi=0.28)$ and EV Sct $(\phi=0.65)$. The EV Sct spectrum is vertically shifted.

points (see Sect. 3.2), and apparently another hypothesis is required to explain such a phenomenon. For this, first of all, it is necessary to find the stars among Cepheids where such a phenomenon is seen, and then to investigate their properties.

Having at our disposal a large data-base of Cepheid highresolution spectra (multiphase observations) collected by us during the past few years, we have searched for visible manifestations of line profile anomalities in these stars. In total, more than 700 spectra of 99 galactic Cepheids were analysed. Part of these spectra were used in galactic metallicity gradient studies (Andrievsky et al. 2002, and references therein), and a description of these spectra can be found in the mentioned above papers. Among the 99 Cepheids we have found four stars, BG Cru, V1334 Cyg, EV Sct and X Sgr, with anomalous line profile features - additional absorption components or unusual asymmetries (hereafter called bumps). They are shown in Figs. 1-5.

Three of them (BG Cru, V1334 Cyg, EV Sct) are s-Cepheids (it is quite possible that X Sgr is also an s-Cepheid, see Sect. 3.4 and Fig. 7). The so-called s-Cepheids (Cepheids with sinusoidal light curves and small amplitudes) are first overtone pulsators. They were discriminated from other classical Cepheids first by qualitative criteria, and then by a more precise quantitative definition, based on the Fourier decomposition of the light curves, was introduced by Antonello et al. (1990). Microlensing surveys (MACHO and EROS) have unambiguously shown that all s-Cepheids pulsate in the first (or second) overtone (Welch et al. 1995; Beaulieu et al. 1995).

The main characteristics of BG Cru, V1334 Cyg, EV Sct and X Sgr are given in Table 1. In this table we also list some properties of a number of other Cepheids that will be discussed in Sect. 3.3.

\section{Possible origin of the detected peculiarity}

As one of the possible origins of the line profile anomalies in the mentioned above Cepheids, non-radial oscillations could be considered.

For years it was believed that non-radial pulsations, being a primary attribute of $\mathrm{B}-\mathrm{A}-\mathrm{F}$ main sequence stars like $\delta$ Sct or $\beta$ Cep pulsating variables, are at the same time not seen (i.e. not excited) in pulsating F-G supergiants (Cepheids, for example). Dziembowski (1971) argued that $p$-wave modes in stars with steep mass concentration towards the center, i.e. in (super)giants, cannot exist because of significant energy dissipation. Osaki (1977) was the first to show that under special conditions, F-G supergiants can be unstable against non-radial oscillations. He considered the atmosphere of a pulsating star as an isolated oscillating zone with a progressive-wave boundary condition at its bottom, and concluded that Cepheid-like stars should be vibrationally unstable not only for radial pulsations, but also for non-radial modes with high spherical harmonic numbers. Osaki (1977) left the question of the actual existence of non-radial pulsation in supergiants to future studies, since up to then no observational evidence of such oscillations was available for Cepheids.

Van Hoolst et al. (1998) considered the possibility of the excitation of non-radial pulsations in classical pulsating stars (Cepheids, RR Lyrae stars, W Vir) by using the RR Lyrae model. They found that a large number of unstable low-degree $(l=1,2)$ modes have frequencies in the vicinity of unstable radial mode frequencies and proposed resonance models to explain the Blazhko effect.

The first strong evidence of the presence of non-radial modes in the line-profile variations of a classical pulsator was reported by Chadid et al. (1999). A detailed frequency analysis based on 669 high resolution spectra of the Blazhko star RR Lyrae clearly revealed the importance of non-linear effects upon the radial fundamental mode, and a multiplet structure with a separation equal to the Blazhko frequency around the main frequency and its harmonics.

It should be noted that classical Cepheid V473 Lyr also demonstrates an unusual amplitude modulation of the light and radial velocity on a time-scale of about 1000 days. Van Hoolst \& Waelkens (1995) have interpreted such behaviour as a resonant interaction between the second overtone and a nonradial mode with approximately the same period (see also Koen 2001).

Another illustrative example among the supergiants is Polaris ( $\alpha$ UMi), a low-amplitude s-Cepheid with a period of 4.0 days. Hatzes \& Cochran (2000) have found a residual component in high-accuracy radial velocities with an amplitude of $400 \mathrm{~m} \mathrm{~s}^{-1}$ and a period of about 40 days. They argue that this is more likely to be due to a non-radial pulsation, rather than to a low-mass companion or to rotational modulation from spots. Non-radial $g$-modes are probably present in the F8Ia supergiant V810 Cen (Kienzle et al. 1998), and in yellow hypergiants like $\rho$ Cas (Lobel et al. 1994).

Butler (1998) presented precision velocity results from a $6 \mathrm{yr}$ survey of 15 supergiants that lie in (or near) the Cepheid instability strip. Periodograms of many of these stars show 
Table 1. Program stars. Cepheids with bumps: upper panel, and additional Cepheids with high $F W H M$ values: lower panel.

\begin{tabular}{|c|c|c|c|c|c|c|}
\hline Star & Type & $P$, day & $<V>$ & $(B-V)$ & $\overline{A_{V}}$ & $F W H M, \AA$ \\
\hline \multicolumn{7}{|c|}{ Cepheids with bumps: } \\
\hline BG Cru & SCEP & 3.3427 & 5.487 & 0.606 & 0.246 & 0.735 \\
\hline V1334Cyg & SCEP & 3.3330 & 5.871 & 0.504 & 0.146 & 0.639 \\
\hline EV Sct & SCEP & 3.0909 & 10.137 & 1.160 & 0.300 & 0.67 : \\
\hline X Sgr & DCEP: & 7.0129 & 4.549 & 0.739 & 0.590 & 0.669 \\
\hline \multicolumn{7}{|c|}{ Other Cepheids with large $F W H M$ values: } \\
\hline FM Aql & DCEP & 6.1142 & 8.270 & 1.277 & 0.724 & 0.647 \\
\hline RX Aur & DCEP & 11.6235 & 7.655 & 1.009 & 0.664 & 0.614 \\
\hline DL Cas & DCEP & 8.0007 & 8.969 & 1.154 & 0.571 & 0.644 \\
\hline SU Cyg & DCEP & 3.8455 & 6.859 & 0.575 & 0.766 & 0.615 \\
\hline Y Lac & DCEP & 4.3238 & 9.146 & 0.731 & 0.705 & 0.622 \\
\hline V340 Nor & SCEP & 11.2870 & 8.375 & 1.149 & 0.310 & 0.653 \\
\hline Y Sgr & DCEP & 5.7734 & 5.744 & 0.856 & 0.725 & 0.609 \\
\hline S Vul & DCEP & 68.464 & 8.962 & 1.892 & 0.588 & 0.601 \\
\hline
\end{tabular}

significant peaks at 50-80 days which are unlikely to be associated with radial pulsation.

\section{Discussion}

\subsection{The bumps}

As it is known, the well-studied non-radial pulsators among B, A-stars show either bumps (they are the common feature of fast rotators, see, for example, Uytterhoeven et al. 2001 for $\kappa$ Sco, Gies \& Kullavanijaya 1988 for $\epsilon$ Per), or some kind of line asymmetry in the slow rotators (Aerts et al. 1994 for $\beta$ Cep stars, Aerts et al. 1999 for Slowly Pulsating B stars).

Figures 1-5 leave no doubt that absorptional peculiarity of the line profiles (bumps) really exists in the spectra of four Cepheids listed in the upper panel of Table 1. For EV Sct, only the blue bump is clearly seen, while both blue and red bumps are visible in the other three Cepheids. This could be due to the small number of EV Sct spectra which were perhaps observed at phases that are not appropriate for simultaneous detection of two bumps. One should note that the bumps are seen on the profiles of all unblended spectral lines. The most prominent bump features and time evolution were detected in X Sgr.

Using the blend separation algorithm of Cassatella (1976), we deconvolved the X Sgr, V1334 Cyg, BG Cru and EV Sct line profiles in order to find the blue/red bump velocity shift relative to a central absorption. For BG Cru and EV Sct we obtained the following $\Delta V_{\mathrm{r}}$ values for the blue bump: $-16.43 \pm$ $0.19 \mathrm{~km} \mathrm{~s}^{-1}$ for BG Cru and $-16.88 \pm 0.14 \mathrm{~km} \mathrm{~s}^{-1}$ (1992), and $-16.40 \pm 0.48 \mathrm{~km} \mathrm{~s}^{-1}$ (2002) for EV Sct.

A sufficient number of spectra of V1334 Cyg and X Sgr, and pronounced bumps, allowed us to resolve phase-dependent changes of the radial velocity of all absorption components in the line profiles. For example, in the case of V1334 Cyg, one can see the flat line bottom as a sum of two equal absorption bumps at $\phi=0.365$, the blue bump at $\phi=0.660$ and the red bumps at $\phi=0.819,0.960,0.997$ (Fig. 3). The radial velocity separation between the displaced absorption bumps and the central absorption component is $+15.1 \mathrm{~km} \mathrm{~s}^{-1}$

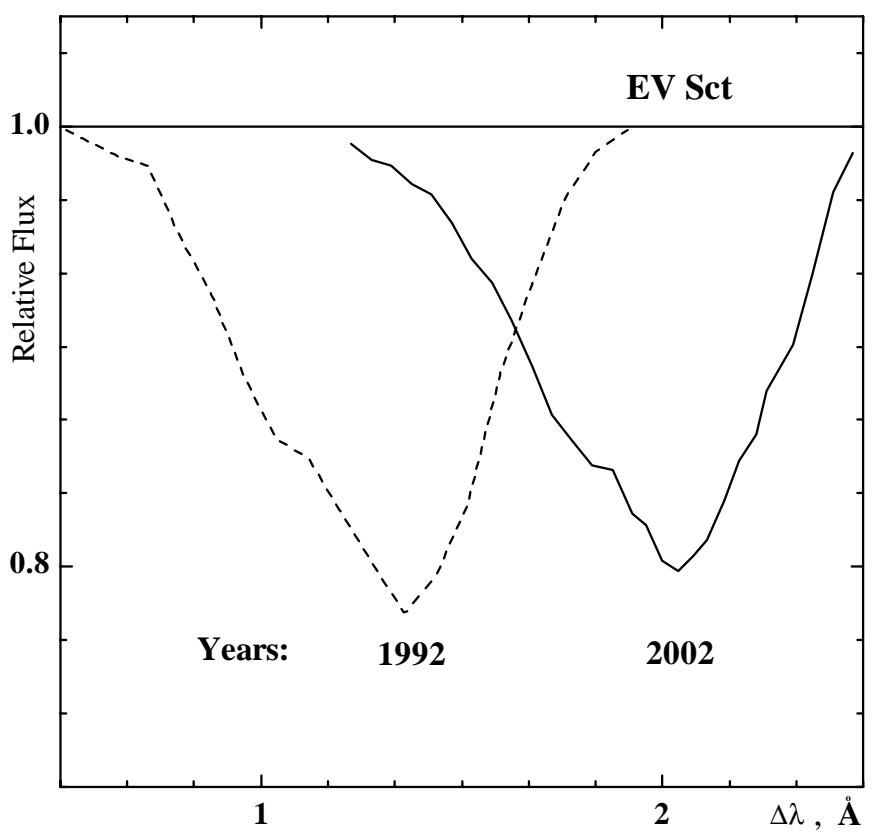

Fig. 2. Averaged line profiles of EV Sct in 1992 and 2002 years. Each profile is a sum of approximately twenty unblended line profiles.

and $-12.4 \mathrm{~km} \mathrm{~s}^{-1}$ at phases 0.819 and 0.365 respectively, but with a large uncertainty of about $\pm 3-4 \mathrm{~km} \mathrm{~s}^{-1}$.

In general, the character of the profile change (transition from blue bump to red one) in V1334 Cyg resembles very much that in the X Sgr spectrum analyzed in this paper (see Figs. 4-5). We have also re-examined the spectra of X Sgr obtained by Luck \& Lambert $(1981,1985)$ in 1978-1979, and find absorption bumps (note that Luck \& Lambert did not consider the multicomponent structure of the line profiles). Thus, one can state that the discussed phenomenon has been persistent for 20 years. Comparison of line profiles from the 1978 and 1997 spectra at similar phases shows an increase of the blue component strength and weakening of the red one. However, this is not a very confident result as the spectra have different resolutions. 


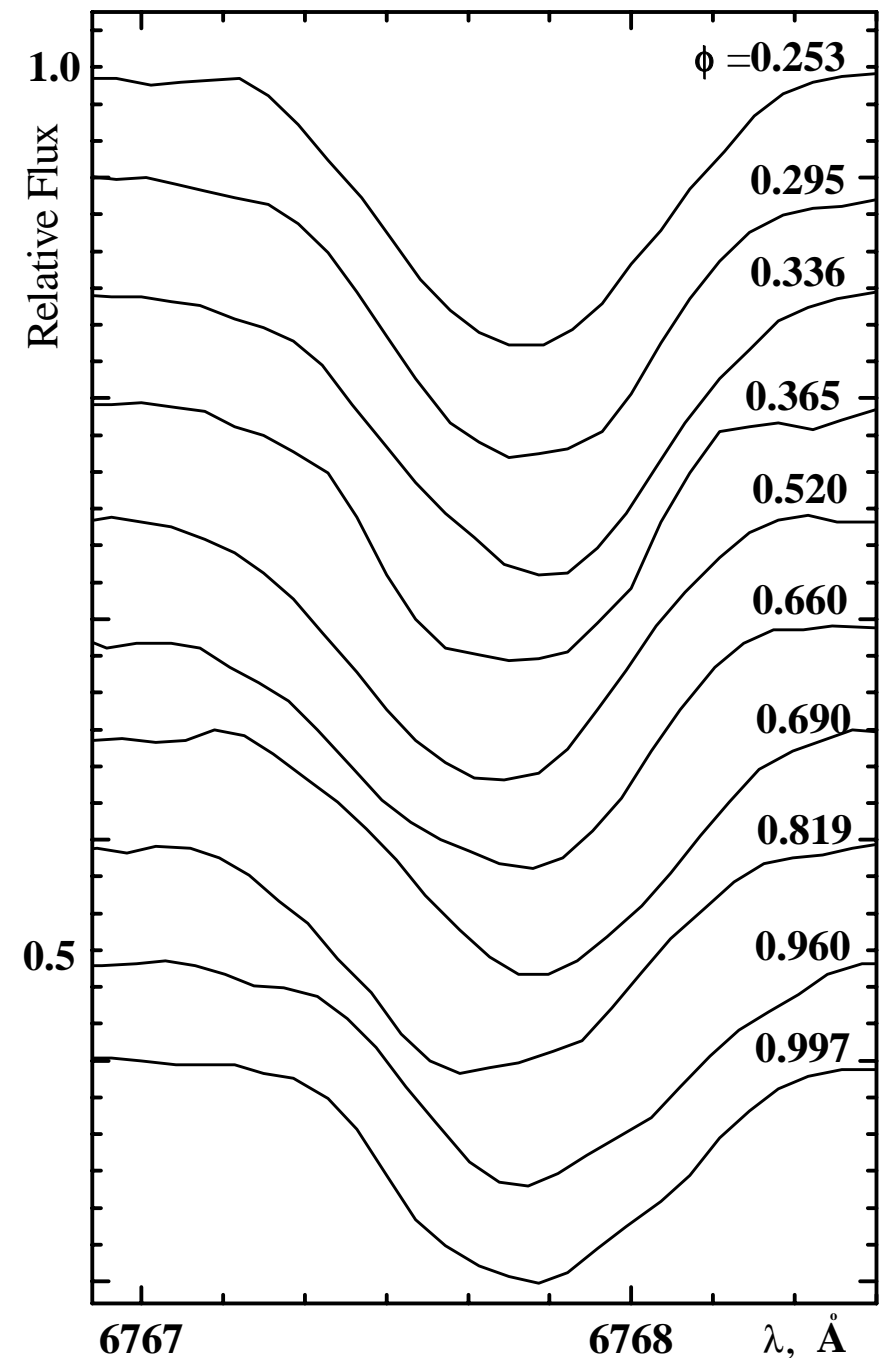

Fig. 3. Observed Ni I $6767.78 \AA$ line profiles in V1334 Cyg spectrum. Phases are indicated near the profiles.

The radial velocity measurements carried out by us, similar to those Sasselov \& Lester (1990), for both satellite absorptions are shown in Fig. 6. The corresponding phases are calculated according to the quadratic elements from Berdnikov \& Ignatova (2000). From Fig. 6 one can conclude that the absorptions are quasi-equidistant in radial velocity space (within the errors of radial velocity measurements which are, nevertheless, rather high). The two satellite absorption features are always shifted at a constant value with respect to the main absorption feature: $+21.3 \pm 1.8 \mathrm{~km} \mathrm{~s}^{-1}$ for the red one and $-24.8 \pm 4.0 \mathrm{~km} \mathrm{~s}^{-1}$ for the blue one, which agree with each other to within the errors of measurement.

The line profile bumps in our four Cepheids resemble those that are seen in line profile of non-radially pulsating stars (e.g. Osaki 1971; Smith 1980; Gies \& Kullavanijaya 1988). According to Shibahashi \& Osaki (1981), the harmonic index $l$ shifts to higher values with increasing $T_{\text {eff }}$. In connection with this, it is interesting to note that our four candidates for non-radial pulsators lie well in the vicinity of the blue edge of the instability strip, as illustrated by Fig. 7. To construct this diagram, the phase-averaged effective temperatures of all our stars were determined following Kovtyukh \& Gorlova (2000). Absolute visual magnitudes were obtained with the help of the "period-luminosity" relation of Gieren et al. (1998).

\subsection{Non-radial pulsation vs. binarity}

In principle, the line splitting of a spectroscopic binary at some phases can mimic a bump feature resulting from non-radial pulsations, and vice versa. If blue and/or red absorption features are detected, but long-term spectroscopic observations are not available, then it is difficult to positively conclude whether one is dealing with a spectroscopic binary star or a non-radial pulsator.

Therefore, it is quite important to search for some independent evidence of the possible spectral binarity of our four Cepheids suspected to be simultaneously the radial and nonradial pulsators.

Berdnikov \& Pastukhova (1995) detected O-C variations for EV Sct with a probable period of $\approx 10000$ days. A large scatter in the light and radial velocity curves has been noted in the work of Pel (1976) and Mermilliod et al. (1987). This could be a sign of EV Sct duplicity. As it was mentioned in the Introduction, Kovtyukh \& Andrievsky detected line splitting in the spectrum of EV Sct, and attributed it to spectroscopic binarity of this Cepheid. The most recent spectral observations of EV Sct, described in the present paper, confirm an existence of the blue bump (Fig. 2). Moreover, both spectra, separated by 10 years but taken at similar pulsation phases, show practically the same velocity difference between the bump and central absorption $\left(-16.88 \mathrm{~km} \mathrm{~s}^{-1}\right.$ and $-16.40 \mathrm{~km} \mathrm{~s}^{-1}$ respectively). This does not favor the hypothesis of spectroscopic binarity, as otherwise, one would expect to see a different velocity between the line components (unless the binary period is very close to 10 years).

Kiss \& Vinko (2000) described the line splitting in V1334 Cyg spectrum, and interpreted such a splitting as a binarity sign. According to Henriksson (1982) V1334 Cyg was known before as a triple star containing an anomalously low-amplitude Cepheid $\left(A_{V}=0.146\right)$, a secondary of B5B8 spectral class $(V=7.9$, at a distance of 0.13 arcsec for epoch 1975.0), and another fainter companion that was detected from the Cepheid's velocity variations on a time-scale of about 5 years (Evans 2000). Nevertheless, both known companions of V1334 Cyg are too faint to contribute significantly to the optical spectrum.

BG Cru is a poorly studied s-Cepheid with a small amplitude $\left(A_{V}=0.203\right)$. Duplicity was suspected on the basis of the low amplitude light variations in the $U$ band (Dean 1981). Szabados (1989) reached the same conclusion using $V_{\gamma}$ variations. Evans (1992) failed to detect a hot companion with IUE data and assigned an upper limit of A1 for the spectral type of the undetected companion.

Evans (1992) provided a lower limit of spectral type A0 for the imputed companion of X Sgr.

Summarizing, the bumps observed in X Sgr, V1334 Cyg, EV Sct and BG Cru spectra are unlikely to be to duplicity (or multiplicity) of these Cepheids. Especially for X Sgr 


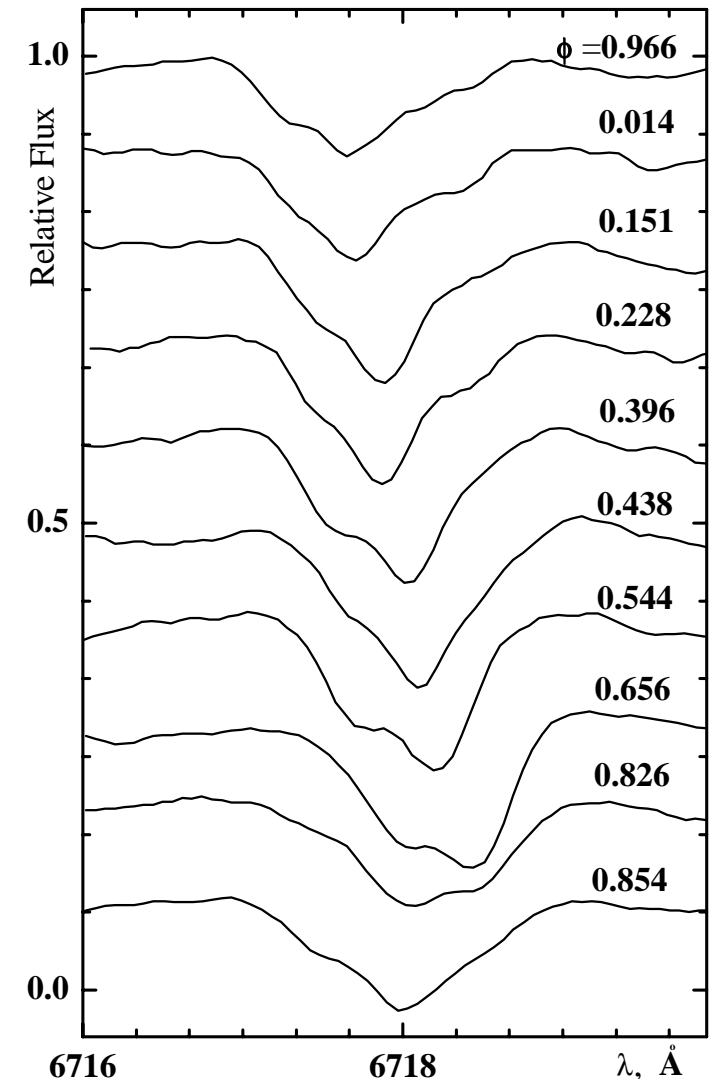

Fig. 4. A sample series of Ca I $6717.69 \AA$ line profiles of X Sgr showing the evolution of the blue to red moving bump structure.

and V1334 Cyg, with a large number of analyzed spectra, the short-time changes of the bump positions do not support the companion-based hypothesis. For BG Cru and EV Sct additional observations are warranted in order to further discriminate between the two possibilities.

\subsection{Other peculiarities of four program Cepheids}

Another interesting feature of our four Cepheids are anomalously broad spectral lines (for V1334 Cyg this was also found by Kiss \& Vinkó 2000, and for EV Sct this was noted by Bersier $\&$ Burki 1996). One should mention that the line $F W H M$ value in Cepheids depends upon the phase, and reflects the effect of a global compression of the atmosphere, as well as the shockwave propagation (Fokin et al. 1996; Bersier \& Burki 1996; Kiss \& Vinkó 2000). For example, Gillet et al. (1999) have used $F W H M$ to trace the turbulent velocity variations in the well known Cepheid $\delta$ Cep. From non-linear and non-adiabatic pulsation models they concluded that the main factor governing the line-broadening processes is the global compression/expansion of the atmosphere, while shock-wave effects turned out to be much weaker.

For the sake of uniformity we have measured and compared the line widths in all available Cepheid spectra at a fixed pulsation phase. The shapes of the lines and their widths vary during the Cepheid pulsation cycle. At the phase of maximum radius the line profiles are highly symmetric due to the absence of systematic atmosphere motions due to pulsation.

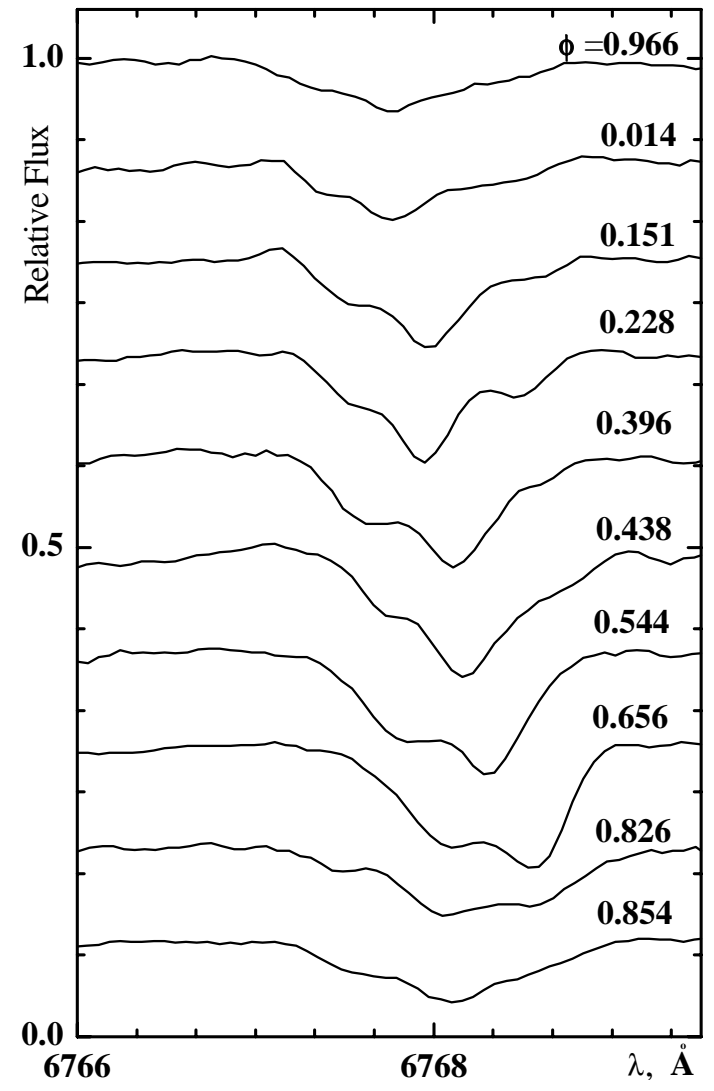

Fig. 5. Same as in Fig. 4 but for Ni I $6767.78 \AA$ line.

This makes the maximum radius phase quite suitable for line width measurements. A similar approach was first used by Bersier \& Burki (1996) for 41 Cepheids (CORAVEL data). Their conclusion was that the value of the equatorial rotational velocity of Cepheids $v_{\text {rot }} \sin i$ is smaller than $10 \mathrm{~km} \mathrm{~s}^{-1}$, and that the line broadening is dominated by the turbulence. Thus, they confirmed the earlier result of Kraft (1966) about very slow rotation of yellow supergiants. The more detailed studies of Fokin et al. (1996) and Takeda et al. (1997) reported for $\delta$ Cep and SU Cas $v_{\text {rot }} \sin i=7.5 \pm 2 \mathrm{~km} \mathrm{~s}^{-1}$ and $v_{\text {rot }} \sin i \simeq$ $0 \mathrm{~km} \mathrm{~s}^{-1}$ respectively (estimates at the phase of near-maximum radius). It should be also noted that Bersier \& Burki excluded from their statistics the following Cepheids: EV Sct, DL Cas and V340 Nor as the stars having the broadest lines. The interpretation of the broadening velocity as rotation is not the only interpretation possible. Macroturbulence would be expected in these stars and Luck \& Lambert $(1981,1985)$ derive Gaussian macroturbulent velocities averaging $8-12 \mathrm{~km} \mathrm{~s}^{-1}$ from profile fits for a number of these stars. At the broadening velocities determined for Cepheids it is impossible to separate a strictly Gaussian macroturbulent profile from a rotation profile.

We have measured the Ca I $6717.687 \AA$ line $F W H M$ for 51 Cepheids at the phase of maximum radius (only for those of our Cepheids the spectra near the phase of a maximum radius are available). The determined $F W H M$ s are given in Table 1 (only for those Cepheids with larger than normal width values), and the histogram of $F W H M$ s is shown in Fig. 8. While Bersier $\&$ Burki (1996) found only three stars with anomalously broad lines, we find 12 stars with $<F W H M>=0.641 \pm 0.038 \AA$ 


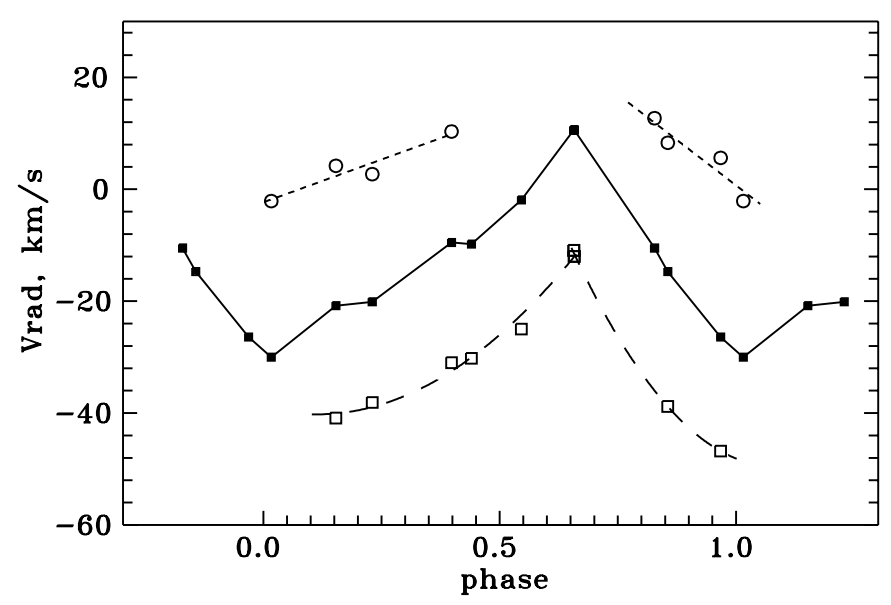

Fig. 6. Radial velocity curves for X Sgr. The filled squares are the velocities of the main absorption component of the line. The bump velocities are shown as open squares (blue one) and open circles (red one).

that are clearly separated from the greater bulk of the Cepheids with $<F W H M>=0.450 \pm 0.034 \AA$. All four of our "bump" stars are in the broad line group.

However, the remaining 8 Cepheids from that group do not show bump features. These Cepheids have different periods (from the 4 "bump" Cepheids) and the only other common feature among them is a small pulsation light amplitude, less than 0.8 in $V$, while Cepheids with narrower lines may have larger amplitudes (Fig. 9). At present we cannot say whether this is a sign of an anti-correlation between pulsation and line width, since for this group it is still unclear which fraction of the line width is contributed by rotation/macroturbulence.

BG Cru has the largest $F W H M$ value, but this maybe simply due to the fact that $\phi=0.282$ (only one spectrum of this star exposed at this phase is available) is not exactly the maximum radius phase, although the lines at this phase appear quite symmetric.

\subsection{Bumps, rotation and resonances}

X Sgr, V1334 Cyg, EV Sct and BG Cru do have broadened lines either due to rotation or macroturbulence, and this probably substantially favors bump detection. Nonetheless, the bumps are not seen in the spectra of the other 8 stars from the group of Cepheids with large FWHM values. What could be the reason for this different behavior? To try to answer this question, one can speculate about the possible connection between the non-radial mode excitation and pulsational resonances in Cepheids. Unfortunately, no detailed study similar to the one of van Hoolst et al. (1998), based upon non-linear non-radial oscillation theory in which the possibility of nonradial mode excitation through resonances, exists for Cepheid models. Most current studies are limited to resonances among radial modes.

Below we briefly summarize the known resonances for Cepheids with periods $P \leq 10^{\mathrm{d}}$ based on the work of Moskalik et al. (1992) and Antonello (1994):

a) There is a well known resonance $F_{2} / F_{0}$ in Cepheids with $P_{0}$ near $10^{\mathrm{d}}$ (see, for example, Moskalik et al. 1992). For

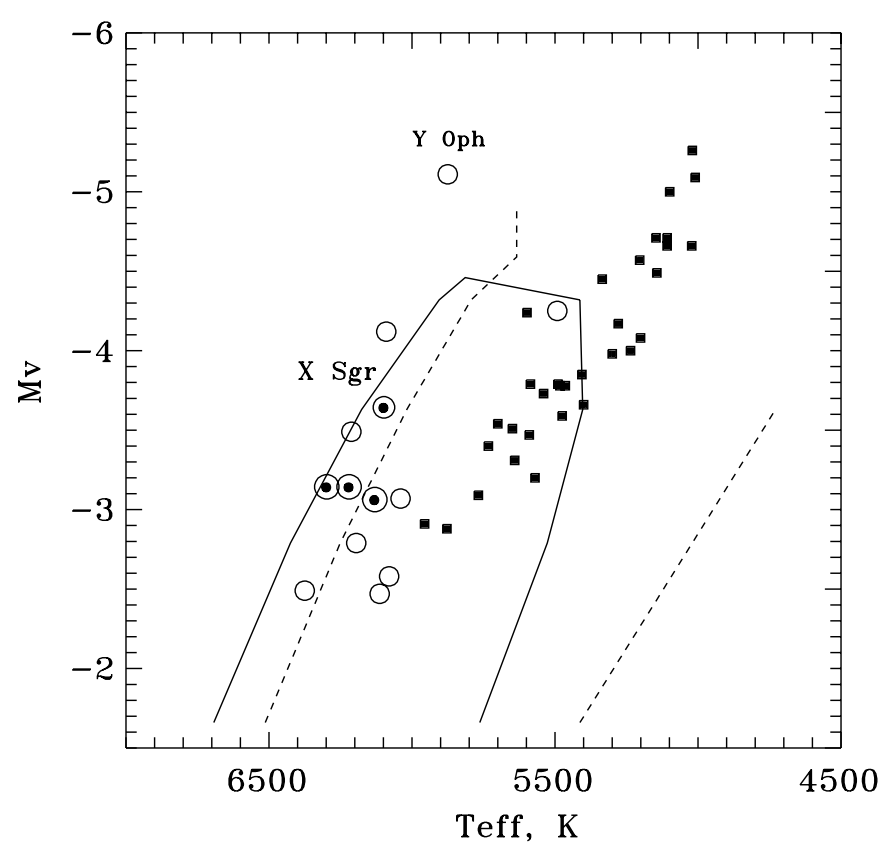

Fig. 7. H-R diagram. The theoretical instability strip for s-Cepheids (1st overtone pulsators) is represented by the solid line, and for fundamental pulsators is marked by the dashed line (turbulent convective Cepheid models by Yecko et al. 1998). Positions of some observed fundamental Cepheids are shown as filled squares and s-Cepheids as circles. The four candidates for non-radial pulsators are shown as dotted circles.

periods in the vicinity of $10^{\mathrm{d}}$, the primary and secondary bumps of the light curve switch roles, and as a result the bump appears to move from the descending to the ascending branch (Hertzsprung progression).

b) The resonance $F_{4} / F_{1}$ near $P_{1}=3.2$ was suspected by Antonello \& Poretti (1986), then noted again by Petersen (1989), and further described by Antonello et al. (1990) in s-Cepheids.

c) The resonance between the fundamental and fourth overtone mode $F_{3} / F_{0}=3$ near $P_{0}=7.5$ has been studied by Moskalik et al. (1992) in their non-linear calculations, and also discussed by Antonello (1994).

It is worth noting that our $3 \mathrm{~s}$-Cepheids, i.e. the first overtone pulsators V1334 Cyg, EV Sct and BG Cru, have their periods $P_{1}$ very close to resonance "b" (although Kienzle et al. 1999 on the basis of the corresponding radial velocity data, suggest that the resonance center lies at a much higher period, closer to 4.6 ).

X Sgr has the longest period (7.0) of the "bump" Cepheids. Resonance "c" could be the origin of the X Sgr bumps. On the other hand, if we suppose that X Sgr is also a first overtone pulsator (this is supported by its position within the HR diagram in Fig. 7), then the period of the unexcited fundamental mode of this star would be $P_{0}=P_{1} / 0.71 \approx 10^{\mathrm{d}}$, which fits case "a".

Interestingly, unlike them, its pulsation amplitude is not the smallest among the Cepheids of the similar periods. We want to point out one more peculiarity of X Sgr - its light curve has broader maximum compared to the Cepheids of similar periods, and a less noticeable secondary bump. We can say that 


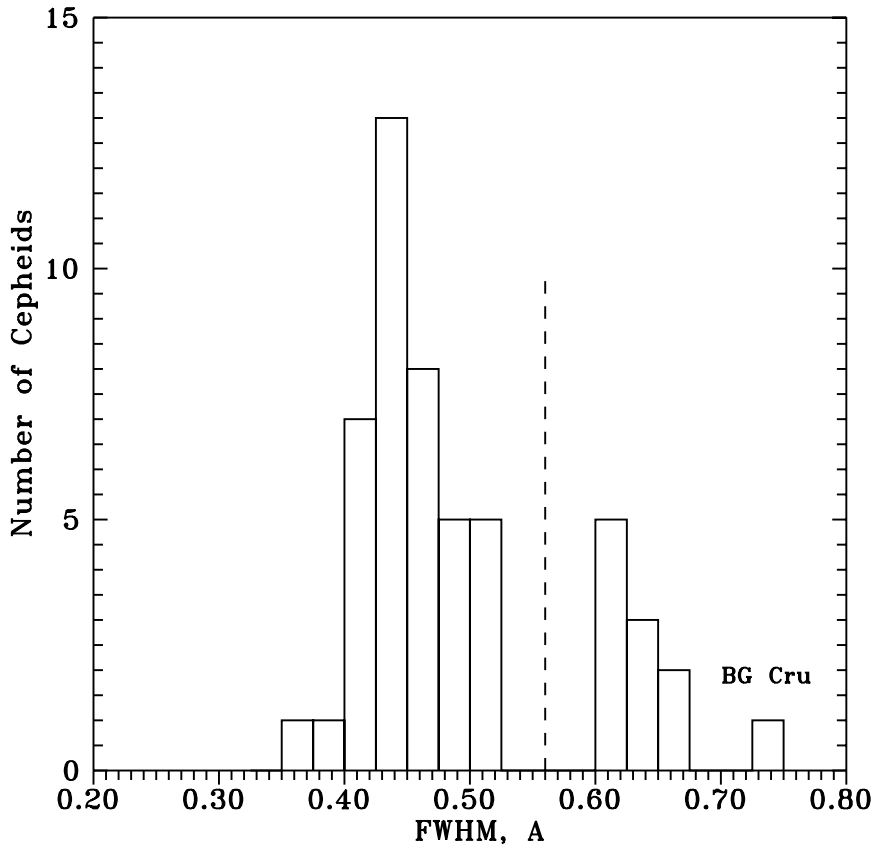

Fig. 8. The distribution of the Cepheid line widths $(F W H M, \AA)$ in the phase of maximum radius is shown. Two groups are clearly separated. $F W H M$ for BG Cru was measured in another phase, which explains its outlying position.

the light curve of X Sgr is intermediate between that of normal Cepheids (fundamental pulsators) and s-Cepheids (pulsating in the 1 st overtone). The abnormal light and radial velocity curves of this Cepheid was first noted by Kovács et al. (1990). That is why it is quite plausible that the star may be in a unique evolutionary stage (switching from one pulsation mode to another, transiting to the bimodal regime, leaving instability strip, etc.), which is also corroborated by the absence of the objects within the resonance period.

\section{Conclusion}

Four Cepheids (BG Cru, EV Sct, V1334 Cyg and X Sgr) are found to have unusual bump features within their spectral lines. They show the following common peculiarities.

- They have periods that are close to resonance values (around 3.2 and 7.5 or $10^{\mathrm{d}}$ ).

- Their light and velocity amplitudes are the smallest among s-Cepheids of similar periods (V1334 Cyg, EV Sct and BG Cru).

- The $F W H M$ values of the spectral lines in these Cepheids are larger than in other ordinary Cepheids.

- Their phase-averaged effective temperatures are rather $\operatorname{high}\left(T_{\text {eff }}>6000 \mathrm{~K}\right)$.

As a preliminary hypothesis, one can suggest that the observed bumps in the line profiles in the spectra of X Sgr, V1334 Cyg, EV Sct and BG Cru can be considered as a combined effect of the rather high line broadening (either due to rotation or macroturbulence), and the non-radial oscillations. Taking into account that fact that characteristic periods of those possible non-radial oscillations are quite close to observed periods of radial pulsations in each stars, one can note that the

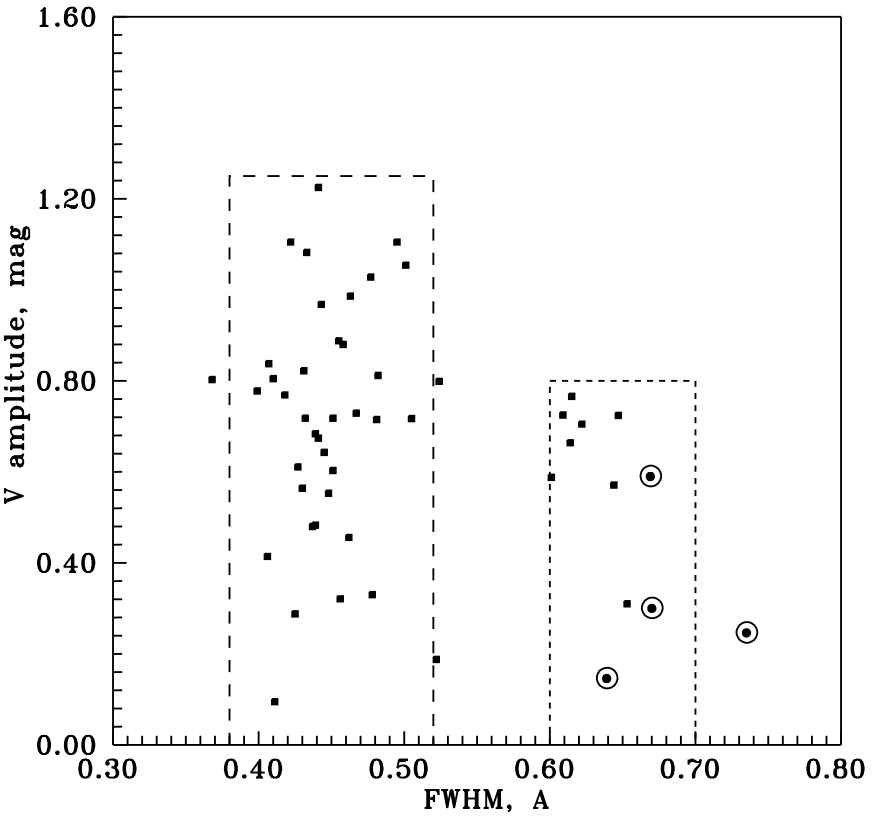

Fig. 9. Light curve amplitudes for two groups of Cepheids with different $F W H M$. One can see that the Cepheids with anomalously broad lines have lower amplitudes $\left(A_{V}<0 \mathrm{~m} 8\right)$. The four candidates for nonradial pulsators are shown as dotted circles.

observed periods of the radial modes in EV Sct, BG Cru, V1334 Cyg (and very likely in X Sgr) are those of the first overtone, and this means that periods of the non-radial modes are shorter than those of the (unexcited) fundamental radial mode, as it should be, for instance, in the case of non-radial $p$-modes. An illustrative example is given by the pulsating yellow supergiant V810 Cen. This star has a period of the fundamental mode $P_{0}=156^{\mathrm{d}}$, and period of non-radial oscillations of about $107^{\mathrm{d}}$. This gives a period ratio of approximately 0.69 (Kienzle et al. 1998), that is close to the corresponding period ratios for our four Cepheids.

Finally, we should note that the detection of the Cepheids having bumps on their spectral line profiles is of particular interest. The proper interpretation of this unusual phenomenon can help 1) to verify the existing pulsational models of Cepheids (this is quite important for the determination of accurate Cepheid masses), and 2) to explain the existence of Cepheids with broadened line profiles, that has no explanation at present.

Acknowledgements. The authors would like to acknowledge Drs. A. Fry, B. Carney, D. Bersier, M. R. Meyer and E. Mamajek for their help obtaining spectral material, Drs. V. Gopka and A. Yushchenko for their version of the Cassatella blend-separation code, and also to Dr. V. Pariev for a very helpful discussion. The authors are indebted to Dr. L. Balona for his very valuable comments, and to the anonymous referee for careful reading of our manuscript and numerous important remarks that helped to improve the paper.

\section{References}

Aerts, C., Waelkens, C., \& De Pauw, M. 1994, A\&A, 286, 136

Aerts, C., De Cat, P., Peeters, E., et al. 1999, A\&A, 343, 872 
Andrievsky, S. M., Kovtyukh, V. V., Luck, R. E., et al. 2002, A\&A, 392, 491

Antonello, E. 1994, A\&A, 282, 835

Antonello, E., \& Poretti, E. 1986, A\&A, 169, 149

Antonello, E., Poretti, E., \& Reduzzi, L. 1990, A\&A, 236, 138

Beaulieu, J.-P., Grison, P., Tobin, W., et al. 1995, A\&A, 303, 137

Berdnikov, L. N., \& Pastukhova, E. N. 1995, SvAstr. Lett., 21, 417

Berdnikov, L. N., \& Ignatova, V. V. 2000, in The Impact of LargeScale Surveys on Pulsating Star Research, ed. L. Szabados, \& D. W. Kurtz, IAU Coll. 176, ASP Conf. Ser., 203, 244

Bersier, D., \& Burki, G. 1996, A\&A, 306, 417

Butler, R. P. 1993, ApJ, 415, 323

Butler, R. P. 1998, ApJ, 494, 342

Butler, R. P., Bell, R. A., \& Hindsley, R. B. 1996, ApJ, 461, 362

Cassatella, A. 1976, A\&A, 48, 281

Chadid, M., Kolenberg, K., Aerts, C., \& Gillet, D. 1999, A\&A, 352, 201

Dean, J. F. 1981, South Afr. Astr. Obs. Circ., No. 6, 10

Dziembowski, W. A. 1971, Acta Astron., 21, 289

Evans, N. R. 1992, ApJ, 384, 220

Evans, N. R. 2000, AJ, 119, 3050

Fokin, A. B., Gillet, D., \& Breitfellner, M. G. 1996, A\&A, 307, 503

Gieren, W. P., Fouqué, P., \& Gómez, M. 1998, ApJ, 496, 17

Gies, D. R., \& Kullavanijaya, A. 1988, ApJ, 326, 813

Gillet, D., Fokin, A. B., Breitfellner, M. G., Mazauric, S., \& Nicolas, A. 1999, A\&A, 344, 935

Hatzes, A. P., \& Cochran, W. D. 2000, AJ, 120, 979

Henriksson, G. 1982, Rep. Obs. Lund., 18, 89

Karp, A. H. 1975a, ApJ, 200, 354

Karp, A. H. 1975b, ApJ, 201, 641

Kienzle, F., Burki, G., Burner, M., \& Meynet, G. 1998, A\&A, 337, 779

Kienzle, F., Moskalik, P., Bersier, D., \& Pont, F. 1999, A\&A, 341, 818
Kiss, L. L., \& Vinkó, J. 2000, MNRAS, 314, 420

Koen, C. 2001, MNRAS, 322, 97

Kovácz, G., Kisvarsányi, E. G., \& Buchler, J. R. 1990, ApJ, 351, 606

Kovtyukh, V. V., \& Andrievsky, S. M. 1999, A\&A, 350, L55

Kovtyukh, V. V., \& Gorlova, N. I. 2000, A\&A, 358, 587

Kraft, R. P. 1956, PASP, 68, 137

Kraft, R. P. 1966, ApJ, 144, 1008

Kraft, R. P. 1967, in Aerodinamic Phenomena in Stellar Atmospheres, ed. R. N. Thomas (New York), IAU Symp., 28, 207

Lobel, A., de Jager, C., Nieuwenhuijzen, H., et al. 1994, A\&A, 291, 226

Luck, R. E., \& Lambert, D. L. 1981, ApJ, 245, 1018

Luck, R. E., \& Lambert, D. L. 1985, ApJ, 298, 782

Mermilliod, J. C., Mayor, M., \& Burki, G. 1987, A\&AS, 70, 389

Moskalik, P., Buchler, J. R., \& Marom, A. 1992, ApJ, 385, 685

Osaki, Y. 1971, PASJ, 23, 485

Osaki, Y. 1977, PASJ, 29, 235

Pel, J. W. 1976, A\&AS, 24, 413

Petersen, J. O. 1989, A\&A, 226, 151

Sasselov, D. D., Lester, J. B., \& Fieldus, M. S. 1989, ApJ, 337, L29

Sasselov, D. D., \& Lester, J. B. 1990, ApJ, 362, 333

Shibahashi, H., \& Osaki, Y. 1981, PASJ, 33, 427

Smith, M. A. 1980, ApJ, 240, 149

Szabados, L. 1989, Commun. Konkoly Obs., No. 94, 1

Takeda, Y., Kawanomoto, S., \& Ando, H. 1997, PASJ, 49, 493

Uytterhoeven, K., Aerts, C., De Cat, P., et al. 2001, A\&A, 371, 1035

van Hoolst, T., \& Waelkens, C. 1995, A\&A, 295, 361

van Hoolst, T., Dziembowski, W. A., \& Kawaler, S. D. 1998, A\&A, 297, 536

Yecko, P. A., Kolláth, Z., \& Buchler, J. R. 1998, A\&A, 336, 553

Welch, D. L., Alcock, C., Bennett, D. P., et al. 1995, in Astrophysical Applications of Stellar Pulsation, ed. R. S. Stobie, \& P. A. Whitelock, IAU Coll. 155, ASP Conf. Ser., 83, 232 